\section{Complete Genome Sequences of Trifolium spp. Inoculant Strains Rhizobium leguminosarum sv. trifolii TA1 and CC275e: Resources for Genomic Study of the Rhizobium-Trifolium Symbiosis}

\author{
Benjamin J. Perry, ${ }^{1}$ Shaun Ferguson, ${ }^{1}$ Aurelie Laugraud, ${ }^{2}$ Steve A. Wakelin, ${ }^{2}$ Wayne Reeve, ${ }^{3}$ and \\ Clive W. Ronson ${ }^{1, \dagger}$ \\ ${ }^{1}$ Department of Microbiology and Immunology, University of Otago, Dunedin 9016, New Zealand \\ ${ }^{2}$ AgResearch, Lincoln Research Centre, Christchurch 8140, New Zealand \\ ${ }^{3}$ College of Science, Health, Engineering and Education, Murdoch University, Perth WA 6150, Australia
}

\section{Abstract}

Rhizobium leguminosarum symbiovar trifolii strains TA1 and CC275e are nitrogen-fixing microsymbionts of Trifolium spp. and have been used as commercial inoculant strains for clovers in pastoral agriculture in Australia and New Zealand. Here we present the complete genome sequences of both strains, resolving their multipartite genome structures and allowing for future studies using genomic approaches.

\section{Genome Announcement}

Rhizobium leguminosarum is a species complex with five genospecies (gs) currently defined based on average nucleotide identity (ANI) (Kumar et al. 2015; Cavassim et al. 2020). Isolates of $R$. leguminosarum symbiovar trifolii engage in nitrogen-fixing endosymbiosis with Trifolium spp. (clover) (Kuykendall et al. 2015). Following a complex molecular dialogue, $R$. leguminosarum sv. trifolii colonizes symbiotic organs on the plant roots, i.e., nodules, wherein the microbes differentiate into organelle-like bacteroids capable of reducing atmospheric dinitrogen into ammonia for assimilation by the plant host (Poole et al. 2018). The $R$. leguminosarum sv. trifolii-Trifolium symbiosis is of significant economic importance as it introduces nitrogen into forage systems used for livestock production (Hoyos-Villegas et al. 2019). R. leguminosarum sv. trifolii CC275e, previously the commercial inoculant of $T$. repens in New Zealand, was replaced in 2005 with strain TA1, which is currently used for T. repens in pastoral agriculture in both Australia and New Zealand, because of ease of manufacturing (Delestre et al. 2015; Drew et al. 2012; Reeve et al. 2013). TA1 was isolated in the 1950s in Tasmania, Australia, from a root nodule of T. subterraneum; CC275e was isolated in Tasmania from a $T$. repens root nodule. Both strains form effective nodules on $T$. repens, but the two strains differ in their symbiotic properties on other agriculturally important clovers. TA1

\section{${ }^{\dagger}$ Corresponding author: C. W. Ronson; Clive.Ronson@otago.ac.nz}

Current address for Shaun Ferguson: Department of Molecular Biology and Genetics, Aarhus University, Aarhus 8000, Denmark

Current address for Steve A. Wakelin: Scion, Rotorua 3010, New Zealand

Benjamin J. Perry and Shaun Ferguson contributed equally to this work.

The author(s) declare no conflict of interest.

Accepted for publication 28 September 2020.
Funding

The work was funded by a University of Otago Research Grant.

\section{Keywords}

genospecies, Rhizobium leguminosarum, replicon, symbiosis, Trifolium 
forms fully effective nodules on $T$. subterraneum but poorly effective nodules on $T$. pratense, while CC275e is fully effective on $T$. pratense but poorly effective on $T$. subterraneum (Delestre et al. 2015; Reeve et al. 2013).

Draft-quality genome assemblies are available for TA1 and CC275e (Reeve et al. 2013; Delestre et al. 2015). The fragmented nature of these assemblies poses problems for use in whole-genome studies utilizing Tn-seq (Perry et al. 2016) or RNA-seq (Green et al. 2019), as portions of their genome sequences may be unrepresented or duplicated. Additionally, R. leguminosarum sv. trifolii genomes are multipartite (Harrison et al. 2010), and this organization is not resolved in draft assemblies. We utilized Oxford Nanopore Technologies (ONT) long-read sequencing and hybrid assembly to resolve the complete genome sequences of TA1 and CC275e.

High-molecular weight DNA was isolated from stationary-phase tryptone yeast broth cultures grown at $28^{\circ} \mathrm{C}$ (Beringer 1974) using a phenol/chloroform extraction method (Meade et al. 1982). Sequencing libraries were prepared using the ONT rapid barcoding kit and

Table 1. Summary of Rhizobium leguminosarum sv. trifolii genomes

\begin{tabular}{|c|c|c|c|c|c|c|c|c|}
\hline Contig & Size (bp) & GC\% & Rh Group & Chromid & Conjugative & Mobilizable & Symbiotic & Accession \\
\hline \multicolumn{9}{|c|}{ Strain TA1, genospecies gsC } \\
\hline Chromosome & $5,036,312$ & 61.0 & & & & & & CP053205.2 \\
\hline pRItTA1A & 498,418 & 58.6 & Rh04 & - & - & + & + & СР053209.2 \\
\hline pRItTA1B & 611,070 & 61.0 & Rh02 & + & - & + & - & СР053208.2 \\
\hline pRItTA1C & 662,289 & 60.6 & Rh03 & - & - & + & - & CP053207.2 \\
\hline pRItTA1D & 805,040 & 60.7 & Rh01 & + & - & + & - & СР053206.2 \\
\hline \multicolumn{9}{|c|}{ Strain CC275e, genospecies gsA } \\
\hline Chromosome & $4,835,120$ & 61.1 & & & & & & СР053439.1 \\
\hline pRItCC275eA & 142,731 & 58.2 & - & - & - & + & - & СР053445.1 \\
\hline pRItCC275eB & 154,225 & 58.1 & Rh06 & - & + & - & + & СР053444.1 \\
\hline pRItCC275eC & 260,097 & 61.1 & Rh03 & - & - & - & - & СР053443.1 \\
\hline pRItCC275eD & 261,950 & 61.0 & Rh05 & - & - & - & - & СР053442.1 \\
\hline pRItCC275eE & 492,830 & 61.4 & Rh02 & + & - & - & - & СР053441.1 \\
\hline pRItCC275eF & 971,705 & 60.3 & Rh01 & + & - & - & - & СР053440.1 \\
\hline
\end{tabular}

A

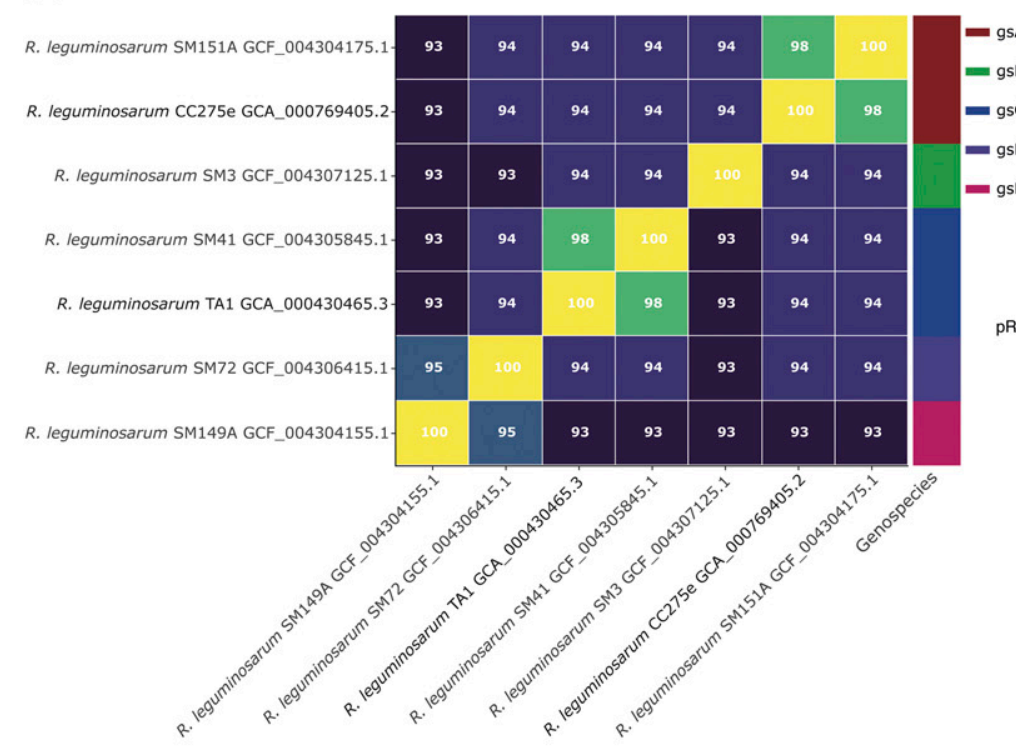

B

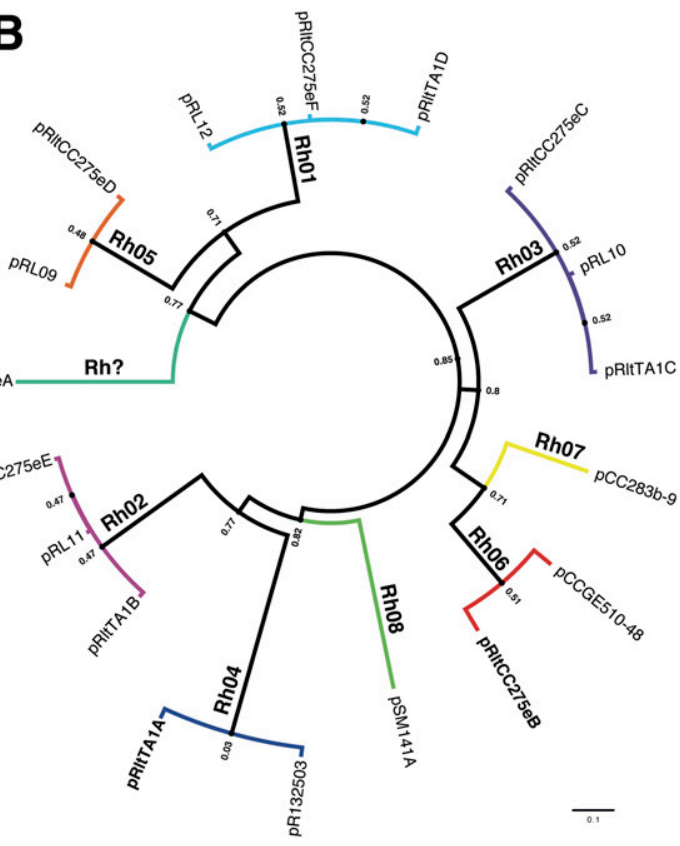

Fig. 1. Genospecies and plasmid Rh group assignments of TA1 and CC275e. A, Average nucleotide identity comparison of TA1 and CC275e genomes with representative strains of the $R$. leguminosarum sv. trifolii species complex outlined by Cavassim et al. (2020). B, Boot-strapped $(1,000)$ neighbor-joining tree of RepABC protein alignment of TA1 and CC275e replicons and replicon Rh group representatives. Symbiotic plasmids of TA1 and CC275e are indicated in bold. 
$500 \mathrm{ng}$ of DNA. Sequencing used a MinION and FLO-MIN106 flowcell (v9.4 Nanopore). Base-calling used guppy v3.4.1 and the template_r9.4.1_450bps_hac.jsn model (Wick et al. 2019). Median read lengths of the TA1 and CC275e ONT reads were 20,774 and 21,124 bp, respectively. Total data generated for TA1 and CC275e equated to $99 \times$ and $61 \times$ coverage, respectively. Hybrid assembly of long-read data and publicly available Illumina data for each genome (Delestre et al. 2015; Reeve et al. 2013) used a previously published pipeline (Perry et al. 2020). The completed genome sequences were deposited in the National Center for Biotechnology Information (NCBI) Genomes database and were annotated with the prokaryotic genome annotation pipeline (Tatusova et al. 2016).

The complete CC275e genome is $7,118,658$ bp in size and contains a circular chromosome and six additional circular replicons (Table 1). CC275e belongs to gs $A$ in the $R$. leguminosarum species complex (Fig. 1A). Alignment of the RepABC proteins from each replicon assigned five of the six replicons to replicon $\mathrm{Rh}$ groups (Fig. 1B; Table 1) previously defined by Cavassim et al. (2020). The symbiotic plasmid pRItCC275eB is the second smallest plasmid in the genome at $154 \mathrm{~kb}$ and carries a VirB/D4-type conjugative system.

The complete TA1 genome is 7,613,129 bp and contains one circular chromosome and four additional circular replicons (Table 1). The completed TA1 genome is $1.0 \mathrm{Mb}$ smaller than the previous draft assembly, due to two large duplications in the previous assembly. TA1 was previously assigned to gsC (Kumar et al. 2015) and this was confirmed here (Fig. 1A). The additional four replicons in the TA1 genome were all assigned to Rh groups (Fig. 1B; Table 1). All non-chromosomal replicons in the TA1 genome harbor homologs of the conjugative relaxase tra $A$, suggesting they are mobilizable; however, none carried genes coding for a conjugative pore, suggesting they are not self-transmissible. The symbiotic plasmid pRItTA1A is the smallest in the genome but is $3.2 \times$ larger than the symbiotic plasmid of CC275e, pRItCC275eB (Table 1).

In summary, the genome structures of both TA1 and CC275e are consistent with the finding of Cavassim et al. (2020), that the R. leguminosarum species complex consists of distinct genospecies, each of which carries a chromosome, two chromids, and a complement of plasmids with $\mathrm{Rh}$ groups characteristic of their genospecies.

Data availability. Long-read sequencing data, complete genome sequences, and annotations can be found under the NCBI Bioproject numbers PRJNA623952 and PRJNA623954 for $R$. leguminosarum sv. trifolii TA1 and $R$. leguminosarum sv. trifolii CC275e, respectively.

\section{Author-Recommended Internet Resource}

Hybrid-Assembly pipeline: https://github.com/BenjaminJPerry/HybridAssembly

\section{Literature Cited}

Beringer, J. E. 1974. R factor transfer in Rhizobium leguminosarum. J. Gen. Microbiol. 84:188-198.

Cavassim, M. I. A., Moeskjær, S., Moslemi, C., Fields, B., Bachmann, A., Vilhjálmsson, B. J., Schierup, M. H., Young, J. P. W., and Andersen, S. U. 2020. Symbiosis genes show a unique pattern of introgression and selection within a Rhizobium leguminosarum species complex. Microb. Genom. 6:e000351.

Delestre, C., Laugraud, A., Ridgway, H., Ronson, C., O'Callaghan, M., Barrett, B., Ballard, R., Griffiths, A., Young, S., Blond, C., Gerard, E., and Wakelin, S. 2015. Genome sequence of the clover symbiont Rhizobium leguminosarum bv. trifolii strain CC275e. Stand. Genomic Sci. 10:121.

Drew, E., Herridge, D., Ballard, R., O'Hara, G., Deaker, R., Denton, M., Yates, R., Gemell, G., Hartley, E., Phillips, L., Seymour, N., Howieson, J., and Ballard, N. 2012. Inoculating Legumes: A Practical Guide. Grains Research \& Development Corporation, Kingston, Australia.

Green, R. T., East, A. K., Karunakaran, R., Downie, J. A., and Poole, P. S. 2019. Transcriptomic analysis of Rhizobium leguminosarum bacteroids in determinate and indeterminate nodules. Microb. Genom. 5:1-16.

Harrison, P. W., Lower, R. P. J., Kim, N. K. D., and Young, J. P. W. 2010. Introducing the bacterial 'chromid': Not a chromosome, not a plasmid. Trends Microbiol. 18: 141-148.
Hoyos-Villegas, V., O'Connor, J. R., Heslop, A. D., Hilditch, A., Jahufer, M. Z. Z., and Barrett, B. A. 2019. Rate of genetic gain for persistence to grazing and dry matter yield in white clover across 90 years of cultivar development. Crop Sci. 59: 537-552.

Kumar, N., Lad, G., Giuntini, E., Kaye, M. E., Udomwong, P., Shamsani, N. J., Young, J. P. W., and Bailly, X. 2015. Bacterial genospecies that are not ecologically coherent: Population genomics of Rhizobium leguminosarum. Open Biol. 5:140133.

Kuykendall, L. D., Young, J. M., Martínez-Romero, E., Kerr, A., and Sawada, H. 2015. Rhizobium. Pages 1-36 in: Bergey's Manual of Systematics of Archaea and Bacteria. Wiley, New York.

Meade, H. M., Long, S. R., Ruvkun, G. B., Brown, S. E., and Ausubel, F. M. 1982. Physical and genetic characterization of symbiotic and auxotrophic mutants of Rhizobium meliloti induced by transposon Tn5 mutagenesis. J. Bacteriol. 149: 114-122.

Perry, B. J., Akter, M. S., and Yost, C. K. 2016. The use of transposon insertion sequencing to interrogate the core functional genome of the legume symbiont Rhizobium leguminosarum. Front. Microbiol. 7:1873.

Perry, B. J., Sullivan, J. T., Colombi, E., Murphy, R. J. T., Ramsay, J. P., and Ronson, C. W. 2020. Symbiosis islands of Loteae-nodulating Mesorhizobium comprise three radiating lineages with concordant nod gene complements and nodulation host-range groupings. Microb. Genom. 6:mgen000426. 
Poole, P., Ramachandran, V., and Terpolilli, J. 2018. Rhizobia: From saprophytes to endosymbionts. Nat. Rev. Microbiol. 16:291-303.

Reeve, W., Tian, R., De Meyer, S., Melino, V., Terpolilli, J., Ardley, J., Tiwari, R., Howieson, J., Yates, R., O'Hara, G., Ninawi, M., Teshima, H., Bruce, D., Detter, C., Tapia, R., Han, C., Wei, C.-L., Huntemann, M., Han, J., Chen, I.-M., Mavromatis, K., Markowitz, V., Ivanova, N., Ovchinnikova, G., Pagani, I., Pati, A., Goodwin, L., Pitluck, S., Woyke, T., and Kyrpides, N. 2013. Genome sequence of the clover-nodulating Rhizobium leguminosarum bv. trifolii strain TA1. Stand. Genomic Sci. 9:243-253.

Tatusova, T., DiCuccio, M., Badretdin, A., Chetvernin, V., Nawrocki, E. P., Zaslavsky, L., Lomsadze, A., Pruitt, K. D., Borodovsky, M., and Ostell, J. 2016. NCBI prokaryotic genome annotation pipeline. Nucleic Acids Res. 44:6614-6624.

Wick, R. R., Judd, L. M., and Holt, K. E. 2019. Performance of neural network basecalling tools for Oxford Nanopore sequencing. Genome Biol. 20:129. 